\title{
A Novel One-pot Synthesis of Isothiocyanates and Cyanamides from Dithiocarbamate Salts Using Environmentally Benign Reagent Tetrapropylammonium Tribromide
}

\author{
Neivotsonuo Bernadette Kuotsu, ${ }^{1,3}$ Latonglila Jamir, ${ }^{2}$ Tovishe Phucho ${ }^{3}$ \\ and Upasana Bora Sinha ${ }^{3, *}$ \\ ${ }^{1}$ Department of chemistry, Kohima Science College (Autonomous), Jotsoma-797002, Nagaland, India \\ ${ }^{2}$ Department of Environmental Science, Nagaland University, Lumami-798627, Nagaland, India \\ ${ }^{3}$ Department of Chemistry, Nagaland University, Lumami-798627, Nagaland, India \\ *Corresponding author: E-mail: upasanabsinha@gmail.com
}

Received: 06-03-2017

\begin{abstract}
A highly efficient and simple protocol for the synthesis of isothiocyanates and cyanamides from their respective amines in the presence of a mild, efficient, and non-toxic reagent tetrapropylammonium tribromide is described. High environmental acceptability of the reagents, cost effectiveness and high yields are the important attributes of this methodology.
\end{abstract}

Keywords: TPATB, desulfurization, oxidation, isothiocyanates, sodium bicarbonate, cyanamide.

\section{Introduction}

Isothiocyanates are one of the most important synthetic intermediates for the preparation of both sulphur and nitrogen containing organic compounds especially for heterocycles. ${ }^{1}$ The isothiocyanate functionality is frequently encountered in natural products, including sesquiterpenes. ${ }^{2}$ Additionally, synthetic isothiocyanates have proven to have some biological activity, such as anti-proliferative $^{3}$ and enzyme inhibitory for the HIV virus. ${ }^{4}$ Numerous methods for the preparation of isothiocyanates have been reported, starting from amines, ${ }^{5}$ dithiocarbamates, ${ }^{6}$ organic halides, ${ }^{7}$ olefins ${ }^{8}$ and aldoximes. ${ }^{9}$ Among the literature methods, the most widely used procedure is the synthesis by the decomposition of dithiocarbamates using heavy metals, ${ }^{10}$ thiophosgene, iodine, ethylchlorocarbamate and claycop. ${ }^{11}$ Although many synthetic methods for the preparation of isothiocyanates have been reported to date, ${ }^{12-32}$ most methods suffer from the employment of highly toxic reagents. Thus, there is still need for a commercially viable and environmentally acceptable protocol for the synthesis of isothiocyanates. We describe herein a modified, environmentally benign and cost-effective met- hod for the synthesis of isothiocyanates via TPATB-mediated decomposition of dithiocarbamate salt in the presence of sodium bicarbonate in water/ethyl acetate biphasic solvent system at room temperature (Scheme 1).

Similarly, in continuation of our work we have synthesised cyanamides from the dithiocarbamate salt. Cyanamides have attracted considerable attention as a class of versatile organic molecules. They have a wide range of uses in organic synthetic chemistry ${ }^{33}$ and coordination chemistry ${ }^{34}$ as they can be used as building blocks for the construction of not only diverse $\mathrm{N}$-containing compounds but also as metal ligands with their unique reactivity and structure of the cyanamide unit. Additionally, it has been found that some cyanamide based compounds show a diversity of interesting bioactivities, inhibition of spontaneous myogenic, and peptide activator activities. ${ }^{35}$ It has also been proved that cyanamide is a natural product present in higher plants even though its distribution is limited. ${ }^{36}$ The broad applications of cyanamides have resulted in the development of several methods for their synthesis over the years. The most common among these is the reaction of cyanogens chloride or bromide with amines or with imide salts. ${ }^{37}$ However, this method involves the use of po- 
tassium or sodium cyanide and bromine for the preparation of cyanogen halide (which is again highly toxic), making the protocol environmentally unacceptable. Literature reports on various other methods for the preparation of cyanamide using different synthetic strategies, such as cyanation of amines using $\mathrm{CN}^{+}$equivalents as synthons, ${ }^{38-44}$ Tiemann rearrangement of amidoximes, ${ }^{45}$ coupling reactions involving $\mathrm{Pd}$ isocyanides, allyl carbonates and trimethylsilyl azide, ${ }^{46}$ and sodium bis(trimethylsilyl)amide as deoxygenating or desulfurizing agents. ${ }^{47}$ Yet another method for the preparation of cyanamides involves the reaction of hypervalent iodine(V) species with $N, N^{1}$-disubstituted glycylamide. ${ }^{48}$ However, all the procedures reported so far seem to have certain environmental concerns as they involve direct or indirect use of toxic and corrosive reagents, strong alkaline conditions, expensive reagents and catalysts, high reaction temperatures and tedious purification procedures. We have been interested to an extent in the synthesis of isothiocyanates and cyanamides, therefore, in this context we develop an alternative method involving the use of alkyl or aryl dithiocarbamate using tetrapropylammonium tribromide as a double desulfurizing agent.

\section{Experimental}

\section{1. General Procedure for the Synthesis of Phenyl Isothiocyanate}

To a stirred and ice cooled suspension of phenyl dithiocarbamate salt (Table 1, compound 1) (540 mg, 2 $\mathrm{mmol})$ in ethylacetate $(5 \mathrm{~mL})$, and water $(5 \mathrm{~mL})$ was added $\mathrm{NaHCO}_{3}$ (336 mg, $4 \mathrm{mmol}$ ). To this was added TPATB (0.852 g, $2 \mathrm{mmol}$ ) pinch wise over a period of 10-15 minutes to yield phenylisothiocyante (Table 1 , compound 1a). During this period, precipitation of elemental sulfur was observed. Completion of the reaction was confirmed by TLC. Ethyl acetate $(10 \mathrm{~mL})$ was further added to the reaction mixture. The organic layer was washed with water (2-5 mL), dried over anhydrous $\mathrm{Na}_{2} \mathrm{SO}_{4}$, concentrated under reduced pressure and purified over a short column of silica gel while eluting it with hexane-ethyl acetate (97:3) to give the pure product (Table 1, compound 1a) (216 mg, 80\%).

\section{2. General Procedure for the Synthesis of Phenylcyanamide}

To a stirred and ice cooled suspension of dithiocarbamate (Table 2, compound 1) $(540 \mathrm{mg}, 2 \mathrm{mmol}$ ) in ethyl acetate $(5 \mathrm{~mL})$, was added $\mathrm{NaHCO}_{3}(336 \mathrm{mg}, 4 \mathrm{mmol})$. To this was then added TPATB $(0.852 \mathrm{~g}, 2 \mathrm{mmol})$ pinch wise over a period of 10-15 minutes to yield phenylisothiocyanate. During this period precipitation of elemental sulfur was observed. After complete addition of TPATB, 25\% aqueous $\mathrm{NH}_{3}(2.5 \mathrm{~mL})$ was added drop wise to the stirred reaction mixture to give 1-phenylthiourea. After stirring for 10 minutes at room temperature, the excess of $\mathrm{NH}_{3}$ was removed in a rotary evaporator whereby the solvent ethyl acetate was also simultaneously removed leaving behind the aqueous layer. To the crude reaction mixture was then further added ethyl acetate $(5 \mathrm{~mL})$ and $\mathrm{NaHCO}_{3}(336 \mathrm{mg}$, $4 \mathrm{mmol})$. To the resultant solution, TPATB $(0.852 \mathrm{~g}, 2$ $\mathrm{mmol}$ ) was added in small pinches, during which further precipitation of elemental sulfur was observed. The conversion of 1-phenylthiourea to phenylcyanamide (Table 2, compound $\mathbf{1 b}$ ) was observed within 5 minutes of the complete addition of TPATB. Completion of the reaction was confirmed by TLC. The precipitated sulfur was filtered, washed with ethyl acetate $(2 \times 5 \mathrm{~mL})$. The organic layer was washed with water $(2 \times 5 \mathrm{~mL})$ and dried over anhydrous $\mathrm{Na}_{2} \mathrm{SO}_{4}$, concentrated under reduced pressure and purified over a short column of silica gel eluting it with hexane-ethyl acetate (97:3) to give the pure product (Table 2, compound $\mathbf{1 b}$ ) (188 $\mathrm{mg}, 80 \%)$ as an oily liquid.

\section{Results and Discussion}

The dithiocarbamic acid salt is readily converted into the corresponding isothiocyanate (Table 1, compound 1a), simply by treating it with TPATB in the presence of sodium bicarbonate in water/ethyl acetate biphasic medium in good to excellent yields in shorter time (15 minutes) as shown in Scheme 1.

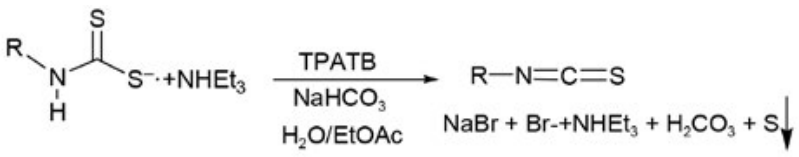

Scheme 1. Preparation of isothiocyanate from dithiocarbamate salt

The use of water/ethyl acetate biphasic solvent system has several potential advantages. In addition to the benign character of both water and ethyl acetate, the coexistence of water with ethyl acetate helps in extracting the isothiocyanate to the organic layer leaving behind the impurities in the aqueous layer which in turn facilitates an easy workup. TPATB is soluble in ethyl acetate and on stirring dissolves, thus getting delivered to the water-ethyl acetate interphase for desulfurization. Moreover, the water phase dissolves the base sodium bicarbonate and retains the dithiocarbamic acid salt in aqueous layer. The use of sodium bicarbonate over organic bases offers a mild and effective green approach towards the synthesis of isothiocyanates. The mechanism of the present transformation is given below in Scheme 2. ${ }^{48}$

Thus, the method provides an ecologically and economically viable process for the preparation of isothiocyanates. The preparation of isothiocyanates was performed 
Table 1. Preparation of Isothiocyanates from Dithiocarbamate Salt and TPATBa

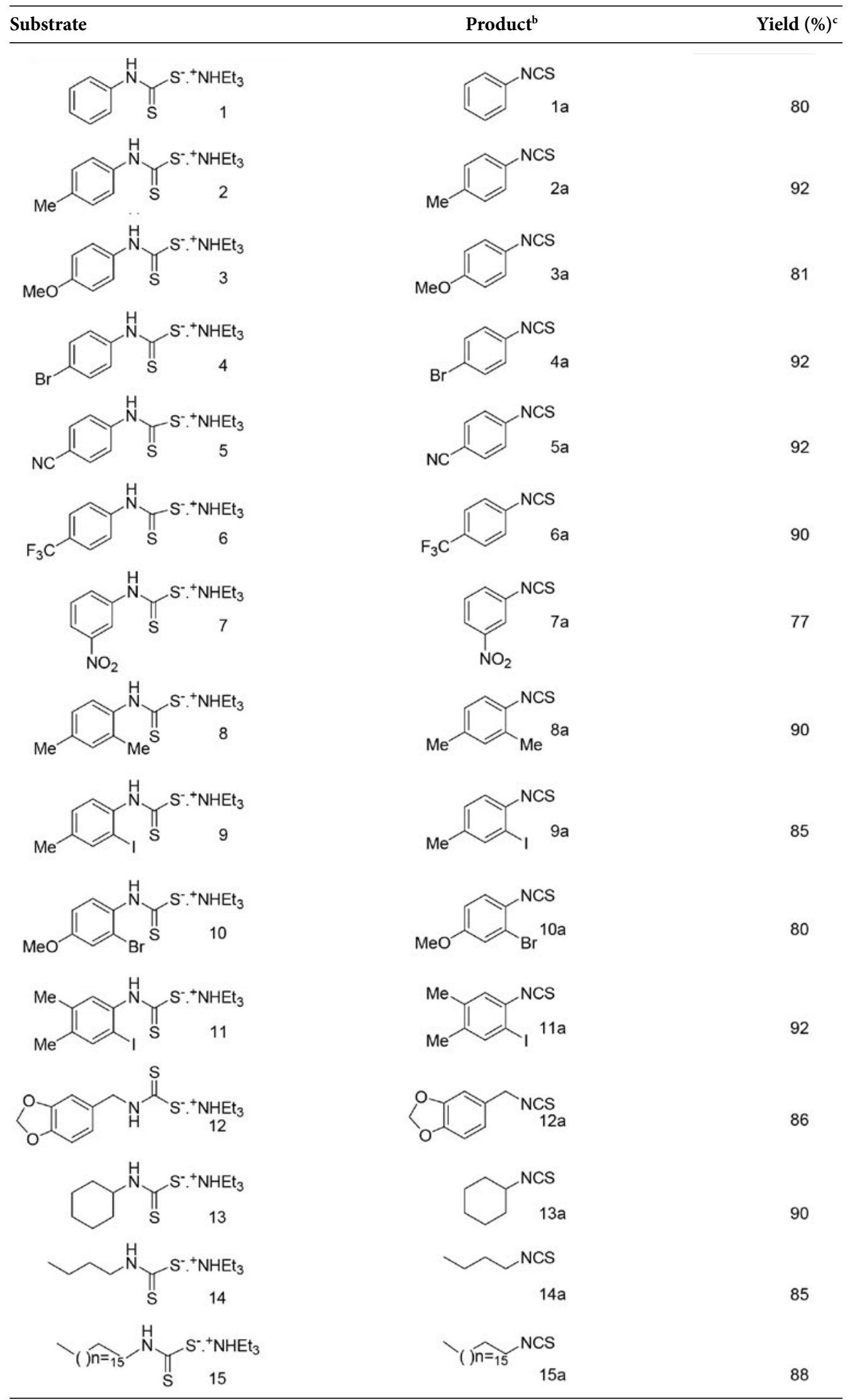

${ }^{\mathrm{a}}$ Reactions were monitored by TLC; ${ }^{\mathrm{b}}$ Confirmed by IR, ${ }^{1} \mathrm{HNMR}$ and ${ }^{13} \mathrm{CNMR} ;{ }^{\mathrm{c}}$ Isolated yield. 


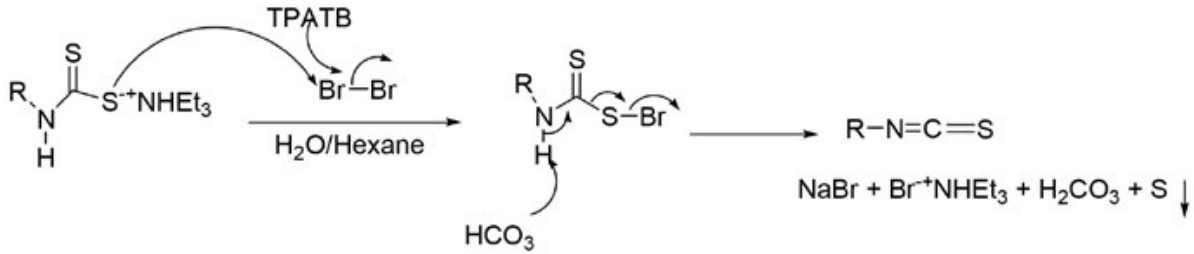

Scheme 2. Mechanism of the formation of isothiocyanate from dithiocarbamate salt

on freshly prepared dithiocarbamate salts synthesized from a variety of structurally different alkyl and aryl amines. The results are summarized in Table 1. Substrates containing activating substituents (Table 1 , compounds 2-3) gave the expected products efficiently as also did substrates containing deactivating substituents (Table 1, compounds 4-7). Trisubstituted substrates (Table 1, compounds 8-10) as well as highly hindered substrates (Table 1, compound 11) gave the corresponding isothiocyanates in high yields. Benzylic substrate (Table 1, compound 12) and aliphatic substrates (Table 1, compounds 13-15) also gave their expected products (Table 1, compound 12a) and (Table 1, compounds 13a-15a) respectively in excellent yields.

Similarly, for the synthesis of cyanamides the methodology was based on: (i) formation of isothiocyanate from alkyl/aryl dithiocarbamate salt by desulfurization with TPATB in the presence of triethylamine as the base in ethyl acetate solvent, (ii) treating the in situ generated isothiocyanate with aqueous $\mathrm{NH}_{3}$ to afford alkyl/aryl thioamides and (iii) further oxidative desulfurization of thioamides to cyanamide with TPATB in the presence of triethylamine (Scheme 2). The mechanism of the present transformation is given below. ${ }^{49}$ Based on these findings, we thus report herein a practical, environmentally benign, high yielding and one pot preparation of cyanamides from dithiacarbamate salts using cheap and non-toxic reagent TPATB (Scheme 3) in an innocuous solvent ethyl acetate.

Implementing this one pot method, a wide variety of aromatic and aliphatic cyanamides have been prepared from their parent dithiocarbamate salts. Phenyl cyanamide (Table 2, compound $\mathbf{1 b}$ ) was obtained in excellent yield from its dithiocarbamate (Table 2, compound $\mathbf{1}$ ). Monosubstituted substrates bearing deactivating substituents (Table 2, compounds 2-6) readily underwent this reaction to produce the desired cyanamides (Table 2, compounds $\mathbf{2 b - 6 b}$ ) in high yields. The method worked smoothly for substrates with electron donating substituents such as (Table 2, compounds 7-8) to afford the corre- sponding cyanamides (Table 2, compounds $\mathbf{7 b}-\mathbf{8 b}$ ) in high yields. A noteworthy aspect is that the present methodology can be applied to substrates containing sensitive substituents such as keto functionality (Table 2, compound 9b) as well as trisubstituted ones (Table 2, compound 10b). Cyclic and open chain aliphatic cyanamides (Table 2, compounds 11b-12b) were obtained from their corresponding dithiocarbamate salts (Table 2, compounds 11-12) under the same reaction conditions. We further investigated the efficacy of this method towards benzylic dithiocarbamate (Table 2, compounds 13-14) and dithiocarbamate salt of homoveratryl amine (Table 2, compound 15) which underwent the reaction smoothly to yield their respective corresponding cyanamide products (Table 2, compounds 13b-14b) and (Table 2, compound 15a) in good yields under the similar reaction conditions.

\section{Spectroscopic and Analytical Data}

1-Isothiocyanato-benzene (1a). Oily; ${ }^{1} \mathrm{H}$ NMR (400 $\left.\mathrm{MHz}, \mathrm{CDCl}_{3}\right) \delta 7.21-7.37$ (m, 5H, ArH). ${ }^{13} \mathrm{C}$ NMR (100 $\left.\mathrm{MHz}, \mathrm{CDCl}_{3}\right) \delta 125.8,127.4,129.6,131.3,135.3 . \mathrm{IR}(\mathrm{KBr})$ 3064, 2164, 2063, 1591, 1489, 1474, 1451, 1070, 927, 905, $749,684 \mathrm{~cm}^{-1}$. Anal. Calcd for $\mathrm{C}_{7} \mathrm{H}_{5} \mathrm{NS}$ (135.19): C, 62.19; H, 3.73; N, 10.36; S, 23.72. Found: C, 62.22; H, 3.71; N, 10.35; S, 23.73.

1-Isothiocyanato-4-methyl-benzene (2a). Oily; ${ }^{1} \mathrm{H}$ NMR $\left(400 \mathrm{MHz}, \mathrm{CDCl}_{3}\right) \delta 2.33\left(\mathrm{~s}, 3 \mathrm{H}, \mathrm{CH}_{3}\right), 7.06-7.13(\mathrm{~m}, 4 \mathrm{H}$, $\mathrm{ArH}) .{ }^{13} \mathrm{C}$ NMR $\left(100 \mathrm{MHz}, \mathrm{CDCl}_{3}\right) \delta 21.2,125.4,128.2$, 130.1, 134.4, 137.4. IR (KBr) 2920, 2094, 1503, 929, 812, 790, $497 \mathrm{~cm}^{-1}$. Anal. Calcd for $\mathrm{C}_{8} \mathrm{H}_{7} \mathrm{NS}$ (149.15): C, 64.36; H, 4.73; N, 9.39; S, 21.51. Found: C, 64.32; H, 4.75; N, 9.41; S, 21.56 .

1-Isothiocyanato-4-methoxy-benzene (3a). Oily; ${ }^{1} \mathrm{H}$ $\operatorname{NMR}\left(400 \mathrm{MHz}, \mathrm{CDCl}_{3}\right) \delta 3.80\left(\mathrm{~s}, 3 \mathrm{H}, \mathrm{CH}_{3}\right), 6.85(\mathrm{~d}, 2 \mathrm{H}$, $J=8.8 \mathrm{~Hz}, \operatorname{Ar} H), 7.16(\mathrm{~d}, 2 \mathrm{H}, J=8.8 \mathrm{~Hz}, \operatorname{ArH}) .{ }^{13} \mathrm{C}$ NMR

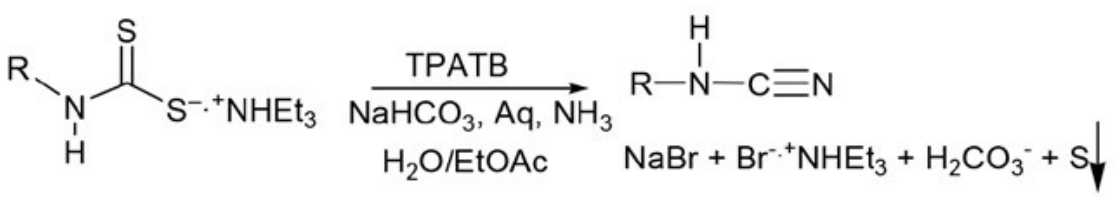

Scheme 3. Mechanism for the formation of cyanamide 
Table 2. Preparation of cyanamides from dithiocarbamates and TPATB ${ }^{\mathrm{a}}$

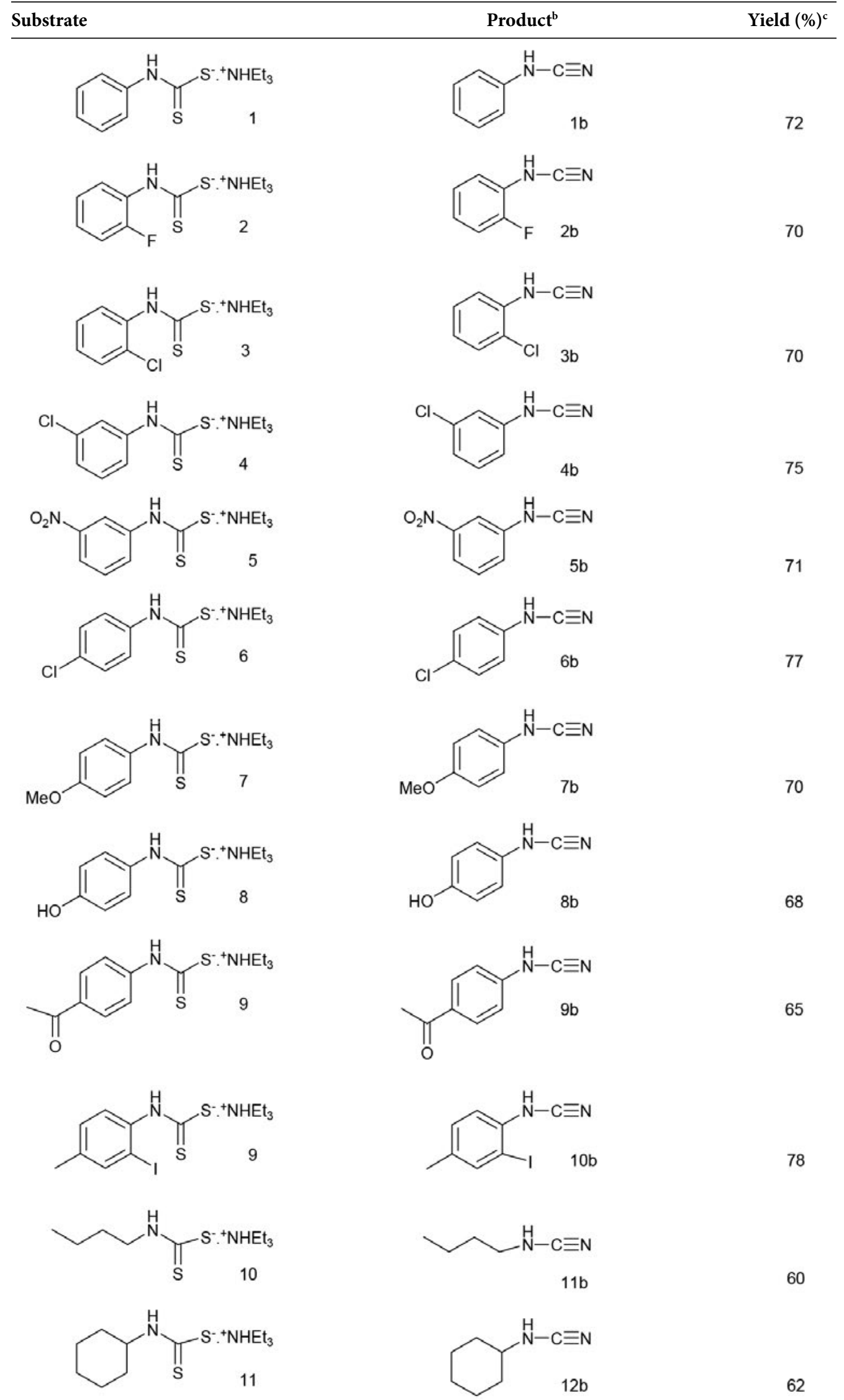

Kuotsu et al.: A Novel One-pot Synthesis of Isothiocyanates ... 
<smiles>CCN[Si]C(=S)NCc1ccccc1</smiles><smiles>CCN=[Sn]C(=S)NCc1ccc2c(c1)OCO2</smiles>

13<smiles>CC=[S]C(=S)NNc1ccc(OC)c(OC)c1</smiles><smiles>N#CNCc1ccccc1</smiles>

$13 \mathrm{~b}$<smiles>N#CNCc1ccc2c(c1)OCO2</smiles><smiles>COc1ccc([14CH2]NC#N)cc1OC</smiles>

64

67

Yield (\%)

66

${ }^{\mathrm{a}}$ Reactions were monitored by TLC; ${ }^{\mathrm{b}}$ Confirmed by IR, ${ }^{1} \mathrm{HNMR}$ and ${ }^{13} \mathrm{CNMR} ;{ }^{\mathrm{c}}$ isolated yieldw

$\left(100 \mathrm{MHz}, \mathrm{CDCl}_{3}\right) \delta 55.4,114.6,123.2,126.8,133.7,158.4$. IR (KBr) 3000, 2956, 2835, 2170, 2098, 1580, 1599, 1503, 1459, 1440, 1292, 1251, 1179, 1166, 1028, 927, 824, 614, $513 \mathrm{~cm}^{-1}$. Anal. Calcd for $\mathrm{C}_{8} \mathrm{H}_{7}$ NOS (165.22): C, 58.16; $\mathrm{H}$, 4.27; N, 8.48; S, 19.40. Found: C, 58.08; H, 4.23; N, 8.34; S, 19.34 .

1-Bromo-4-isothiocyanato-benzene (4a). White solid, m.p. $58^{\circ} \mathrm{C}$ (lit. $\left.{ }^{\text {la }} 58^{\circ} \mathrm{C}\right),{ }^{1} \mathrm{H}$ NMR $\left(400 \mathrm{MHz}, \mathrm{CDCl}_{3}\right) \delta 7.09$ $(\mathrm{d}, 2 \mathrm{H}, J=8.8 \mathrm{~Hz}, \mathrm{ArH}), 7.47(\mathrm{~d}, 2 \mathrm{H}, J=8.8 \mathrm{~Hz}, \mathrm{ArH}) .{ }^{13} \mathrm{C}$ NMR (100 MHz, $\left.\mathrm{CDCl}_{3}\right) \delta 120.8,127.2,130.5,132.8$, 136.9. IR (KBr) 3074, 2925, 2171, 2071, 1578, 1478, 1474, 1399, 1067, 1011, 923, 818, 490, $438 \mathrm{~cm}^{-1}$. Anal. Calcd for $\mathrm{C}_{7} \mathrm{H}_{4} \mathrm{BrNS}$ (214.03): C, 39.24; H, 1.88; N, 6.54; S, 14.99. Found: C, 39.21; H, 1.93; N, 6.50; S, 15.04.

4-Isothiocyanato-benzonitrile (5a). White solid; m.p. 119-120 ${ }^{\circ} \mathrm{C}\left(\right.$ lit. $\left.^{21} 121^{\circ} \mathrm{C}\right),{ }^{1} \mathrm{H}$ NMR $\left(\mathrm{CDCl}_{3}, 400 \mathrm{MHz}\right) \delta$ $7.31(\mathrm{~d}, J=8.8 \mathrm{~Hz}, 2 \mathrm{H}), 7.66(\mathrm{~d}, J=8.8 \mathrm{~Hz}, 2 \mathrm{H}) ;{ }^{13} \mathrm{C} \mathrm{NMR}$ $\left(\mathrm{CDCl}_{3}, 100 \mathrm{MHz}\right) \delta 110.6,117.9,126.5,133.6,135.9$, 139.4; IR (KBr) 3435, 2197, 2124, 2104, 1591, 1492, 1277, 933, 836, $544 \mathrm{~cm}^{-1}$. Anal. Calcd for $\mathrm{C}_{8} \mathrm{H}_{4} \mathrm{~N}_{2} \mathrm{~S}$ (166.13): C, 59.95; H, 2.51; N, 17.49; S, 20.03. Found: C, 59.98; H, 2.49; N, 17.45; S, 19.98 .

1-Isothiocyanato-4-trifluoromethyl-benzene (6a). White solid; m.p. $43{ }^{\circ} \mathrm{C}$ (lit. ${ }^{22}$ bp $\left.205-207^{\circ} \mathrm{C}\right) ;{ }^{1} \mathrm{H}$ NMR $\left(\mathrm{CDCl}_{3}\right.$, $400 \mathrm{MHz}) \delta 7.32(\mathrm{~d}, J=8.4 \mathrm{~Hz}, 2 \mathrm{H}, \mathrm{ArH}), 7.61(\mathrm{~d}, J=8.4$ $\mathrm{Hz}, 2 \mathrm{H}, \mathrm{ArH}) ;{ }^{13} \mathrm{C} \mathrm{NMR}\left(\mathrm{CDCl}_{3}, 100 \mathrm{MHz}\right) \delta 122.4,125.1$, $126.1,126.9,127.0,129.0,129.4,135.15,138.4$; IR (KBr) 3427, 2081, 1613, 1413, 1325, 1137, 1106, 1066, 839, 590 $\mathrm{cm}^{-1}$. Anal. Calcd for $\mathrm{C}_{8} \mathrm{H}_{4} \mathrm{~F}_{3} \mathrm{NS}$ (203.12): C, 47.26; $\mathrm{H}, 1.98$; N, 6.89; S, 15.79. Found: C, 47.22; H, 1.96; N, 6.91; S, 15.75.

1-Isothiocyanato-3-nitro-benzene (7a). Oily; ${ }^{1} \mathrm{H}$ NMR $\left(400 \mathrm{MHz} \mathrm{CDCl}_{3}\right) \delta 7.54(\mathrm{~s}, 2 \mathrm{H}, \mathrm{ArH}), 8.06$ (s, 1H, ArH),
8.11-8.14 (m, 1H, ArH). ${ }^{13} \mathrm{C}$ NMR (100 MHz, $\left.\mathrm{CDCl}_{3}\right) \delta$ $120.7,121.9,130.6,131.6,133.3,139.6,148.8$. IR (KBr) 3091, 3074, 2227, 2161, 2106, 1526, 1470, 1348, 1302, 892, $809,736,665 \mathrm{~cm}^{-1}$. Anal. Calcd for $\mathrm{C}_{7} \mathrm{H}_{4} \mathrm{~N}_{2} \mathrm{O}_{2} \mathrm{~S}$ (180.13): C, 46.63; H, 2.23; N, 15.55; S, 17.81. Found: C, 46.65; H 2.26; $\mathrm{N}, 15.51 ; \mathrm{S}, 17.78$.

1-Isothiocyanato-2,4-dimethyl-benzene (8a). Oily; ${ }^{1} \mathrm{H}$ NMR $\left(400 \mathrm{MHz} \mathrm{CDCl}_{3}\right) \delta 2.30\left(\mathrm{~s}, 3 \mathrm{H}, \mathrm{CH}_{3}\right), 2.33(\mathrm{~s}, 3 \mathrm{H}$, $\left.\mathrm{CH}_{3}\right), 6.96(\mathrm{~d}, 1 \mathrm{H}, J=9.2 \mathrm{~Hz}, \mathrm{ArH}), 7.01(\mathrm{~s}, 1 \mathrm{H}, \mathrm{ArH}), 7.07$ $(\mathrm{d}, 1 \mathrm{H}, J=8.0 \mathrm{~Hz}, \mathrm{ArH}) .{ }^{13} \mathrm{C}$ NMR $\left(100 \mathrm{MHz} \mathrm{CDCl}_{3}\right) \delta$ $18.2,21.1,125.6,127.4,131.2,134.6,137.4$. IR (KBr) 2920, 2131, 2085, 1490, 1455, 1379, 1229, 1036, 947, 901, 875, $812 \mathrm{~cm}^{-1}$. Anal. Calcd for $\mathrm{C}_{9} \mathrm{H}_{9} \mathrm{NS}$ (163.17): C, 66.19; $\mathrm{H}$, $5.55 ; \mathrm{N}, 8.58 ; \mathrm{S}, 19.66$. Found: C, 66.15; H, 5.52; N, 8.63; S, 19.64 .

2-Iodo-1-isothiocyanato-4-methylbenzene (9a). White solid; m.p. 62-65 ${ }^{\circ} \mathrm{C}$; ${ }^{1} \mathrm{H} \mathrm{NMR}\left(400 \mathrm{MHz}, \mathrm{CDCl}_{3}\right) \delta 2.30$ (s, $\left.3 \mathrm{H}, \mathrm{CH}_{3}\right), 7.13(\mathrm{~m}, 2 \mathrm{H}, \mathrm{ArH}), 7.62(\mathrm{~s}, 1 \mathrm{H}, \mathrm{ArH}) .{ }^{13} \mathrm{C} \mathrm{NMR}$ $\left(100 \mathrm{MHz} \mathrm{CDCl}_{3}\right) \delta 20.9,94.2,126.7,130.1,132.3,136.1$, 139.1, 139.9. IR (KBr) 2916, 2134, 1633, 1474, 1042, 929, $811 \mathrm{~cm}^{-1}$.

2-Bromo-1-isothiocyanato-4-methoxy-benzene (10a). White solid; m.p. $77^{\circ} \mathrm{C}$; ${ }^{1} \mathrm{H}$ NMR $\left(400 \mathrm{MHz}, \mathrm{CDCl}_{3}\right) \delta$ $3.80\left(\mathrm{~s}, 3 \mathrm{H}, \mathrm{CH}_{3}\right), 6.82(\mathrm{~m}, 1 \mathrm{H}, \mathrm{ArH}), 7.11(\mathrm{~m}, 1 \mathrm{H}, \mathrm{ArH})$, $7.18(\mathrm{~d}, 1 \mathrm{H}, J=8.0 \mathrm{~Hz}, \mathrm{ArH}) ;{ }^{13} \mathrm{C} \mathrm{NMR}\left(100 \mathrm{MHz} \mathrm{CDCl}_{3}\right)$ $\delta 56.0,114.4,118.5,121.6,124.1,127.7,136.7,158.8$; IR (KBr) 2972, 2125, 1594, 1560, 1483, 1296, 1263, 1220, 1039, 807, $617 \mathrm{~cm}^{-1}$. Anal. Calcd for $\mathrm{C}_{8} \mathrm{H}_{6} \operatorname{BrNOS}(244.05)$ : C, 39.33; H, 2.47; N, 5.73; S, 3.14. Found: C, 39.29; H, 2.52; $\mathrm{N}, 5.70 ; \mathrm{S}, 3.11$.

1-Iodo-2-isothiocyanato-4,5-dimethyl-benzene (11a). White solid; m.p. $54{ }^{\circ} \mathrm{C} ;{ }^{1} \mathrm{H} \mathrm{NMR}\left(400 \mathrm{MHz}, \mathrm{CDCl}_{3}\right) \delta 2.18$ 
(s, 3H, $\left.\mathrm{CH}_{3}\right), 2.22\left(\mathrm{~s}, 3 \mathrm{H}, \mathrm{CH}_{3}\right), 6.99$ (s, 1H, ArH), 7.30 (s, $1 \mathrm{H}, \mathrm{ArH}) ;{ }^{13} \mathrm{C} \mathrm{NMR}\left(100 \mathrm{MHz}, \mathrm{CDCl}_{3}\right) \delta 19.4,19.5,117.2$, $124.1,127.8,129.3,133.8,137.4,137.9$; IR (KBr) 2922, 2853, 2192, 2118, 1472, 1372, 1273, 1076, 1048, 933, 882, 824, $708 \mathrm{~cm}^{-1}$. Anal. Calcd for $\mathrm{C}_{9} \mathrm{H}_{8}$ INS: C, 37.39; H, 2.79; N, 4.84; S, 11.09. Found: C, 37.42; H, 2.81; N, 4.81; S, 11.06. MS ( $\left.\mathrm{AP}^{+}\right)$Calcd for $\mathrm{C}_{9} \mathrm{H}_{8} \mathrm{INS}$ : 288.94. Found: $288.97\left(\mathrm{M}^{+}\right)$.

5-(Isothiocyanatomethyl)benzo $[d][1,3]$ dioxole (12a). Reddish oil. ${ }^{1} \mathrm{H}$ NMR (400 $\left.\mathrm{MHz}, \mathrm{CDCl}_{3}\right) \delta 4.59(\mathrm{~s}, 2 \mathrm{H}$, $\left.\mathrm{CH}_{2}\right), 5.98\left(\mathrm{~s}, 2 \mathrm{H}, \mathrm{CH}_{2}\right), 6.74-6.80(\mathrm{~m}, 3 \mathrm{H}, \mathrm{ArH}) .{ }^{13} \mathrm{C} \mathrm{NMR}$ $\left(100 \mathrm{MHz} \mathrm{CDCl}_{3}\right) \delta 48.7,101.5,107.7,108.6,120.7,128.0$, $132.1,147.8,148.2$. IR (KBr) 2895, 2087, 1503, 1445, 1369, $1322,1251,1101,1028,924 \mathrm{~cm}^{-1}$.

Isothiocyanato-cyclohexane (13a). ${ }^{1} \mathrm{H}$ NMR (400 MHz, $\left.\mathrm{CDCl}_{3}\right) \delta 1.28-1.96\left(\mathrm{~m}, 10 \mathrm{H}, \mathrm{CH}_{2}\right), 3.67(\mathrm{~m}, 1 \mathrm{H}, \mathrm{CH}) .{ }^{13} \mathrm{C}$ NMR (100 MHz, $\left.\mathrm{CDCl}_{3}\right) \delta 23.0,24.9,33.0,55.2,129.6$. IR (KBr) 2937, 2858, 2175, 2102, 2060, 1450, 1361, 1320, 986, 891, 720, $702 \mathrm{~cm}^{-1}$. Anal. Calcd for $\mathrm{C}_{7} \mathrm{H}_{11} \mathrm{NS}$ (141.23): C, 59.53; H, 7.85; N, 9.92; S, 22.70. Found: C, 59.50; H, 7.81; N, 9.88; S, 22.74 .

1-Isothiocyanato-n-butane (14a). Oily liquid. ${ }^{1} \mathrm{H}$ NMR $\left(400 \mathrm{MHz} \mathrm{CDCl}_{3}\right) \delta 0.92\left(\mathrm{t}, J=7.4 \mathrm{~Hz}, 3 \mathrm{H}, \mathrm{CH}_{3}\right), 1.37-$ $1.47\left(\mathrm{~m}, 2 \mathrm{H}, \mathrm{CH}_{2}\right), 1.61-1.69\left(\mathrm{~m}, 2 \mathrm{H}, \mathrm{CH}_{2}\right), 3.42(\mathrm{t}, J=6.6$ $\left.\mathrm{Hz}, 2 \mathrm{H}, \mathrm{CH}_{2}\right) .{ }^{13} \mathrm{C} \mathrm{NMR}\left(100 \mathrm{MHz}, \mathrm{CDCl}_{3}\right) \delta 13.2,19.7$, $31.9,44.7,129.4$. IR (KBr) 2925, 2088. 1597, 1401, 1218, $1116,753 \mathrm{~cm}^{-1}$.

1-Isothiocyanato-octadecane (15a). Gummy, ${ }^{1} \mathrm{H}$ NMR $\left(\mathrm{CDCl}_{3}, 400 \mathrm{MHz}\right) \delta 0.879\left(\mathrm{t}, J=6.8 \mathrm{~Hz}, 3 \mathrm{H}, \mathrm{CH}_{3}\right), 1.25$ $\left(\mathrm{m}, 28 \mathrm{H}, \mathrm{CH}_{2}\right), 1.71-1.72\left(\mathrm{~m}, 4 \mathrm{H}, \mathrm{CH}_{2}\right), 3.50(\mathrm{t}, J=6.4 \mathrm{~Hz}$, $\left.2 \mathrm{H}, \mathrm{CH}_{2}\right) ;{ }^{13} \mathrm{C} \mathrm{NMR}\left(\mathrm{CDCl}_{3}, 100 \mathrm{MHz}\right) \delta 14.3,22.8,26.7$, 29.0, 29.5, 29.7, 29.8, 30.1, 32.1, 45.2; IR (KBr) 2923, 2853, 2185, 2096, 1463, 1455, 1346, $721 \mathrm{~cm}^{-1}$. Anal. Calcd for $\mathrm{C}_{19} \mathrm{H}_{37} \mathrm{NS}$ : C, 73.24; H, 11.97; N, 4.50; S, 10.29. Found: C, 73.27; H, 12.01; N, 4.48; S, 10.25. MS (ES-) Calcd for $\mathrm{C}_{19} \mathrm{H}_{37} \mathrm{NS}$ : 311.26 . Found: $311.19\left(\mathrm{M}^{+}\right)$.

Phenyl cyanamide (1b). Gummy; ${ }^{1} \mathrm{H}$ NMR $\left(\mathrm{CDCl}_{3}, 400\right.$ $\mathrm{MHz}) \delta 7.02-7.07$ (m, 3H, ArH), 7.28-7.33 (m, 2H, ArH), 7.64 (brs, $1 \mathrm{H}, \mathrm{NH}) .{ }^{13} \mathrm{C} \mathrm{NMR}\left(100 \mathrm{MHz}, \mathrm{CDCl}_{3}\right) \delta 112.2$, $115.5,123.6,129.8,137.4$. IR (KBr) 3175, 2919, 2227, 1600, $1501,1249,748,689 \mathrm{~cm}^{-1}$. Anal. Calcd for $\mathrm{C}_{7} \mathrm{H}_{6} \mathrm{~N}_{2}(118.13)$ : C, 71.17; H, 5.12; N, 23.71. Found: C, 71.27; H, 5.09; N, 23.67 .

2-Fluorophenyl cyanamide (2b). White solid; m.p. $95^{\circ} \mathrm{C}$; ${ }^{1} \mathrm{H} \mathrm{NMR}\left(\mathrm{CDCl}_{3}, 400 \mathrm{MHz}\right) \delta 6.87$ (brs, 1H, NH), 6.907.45 (m, 4H, ArH). $\left.{ }^{13} \mathrm{C} \mathrm{NMR} \mathrm{(100} \mathrm{MHz,} \mathrm{CDCl}_{3}\right) \delta 110.9$, $115.7,115.9,116.8,124.1,124.1125 .09,125.12,125.6$, $125.8,150.1,152.5$. IR (KBr) 3068, 2037, 1606, 1587, 1495, $1265,1212,1104,941,808,752 \mathrm{~cm}^{-1}$. Anal. Calcd for $\mathrm{C}_{7} \mathrm{H}_{5} \mathrm{FN}_{2}$ (136.13): C, 61.76; H, 3.70; N, 20.58. Found: C, $61.80 ; \mathrm{H}, 3.73 ; \mathrm{N}, 23.53$.
2-Chlorophenyl cyanamide (3b). White solid; m.p. 101$103{ }^{\circ} \mathrm{C} ;{ }^{1} \mathrm{H}$ NMR $\left(\mathrm{CDCl}_{3}, 400 \mathrm{MHz}\right) \delta 6.56$ (brs, $\left.1 \mathrm{H}, \mathrm{NH}\right)$, 7.05 (m, 1H, ArH), 7.31 (m, 2H, ArH), 7.35 (m, 1H, ArH). ${ }^{13} \mathrm{C}$ NMR $\left(100 \mathrm{MHz}, \mathrm{CDCl}_{3}\right) \delta 110.0,116.2,120.4,124.5$, 128.6, 129.9, 134.3. IR (KBr) 3163, 2921, 2243, 1598, 1500, $1426,1295,1049 \mathrm{~cm}^{-1}$. Anal. Calcd for $\mathrm{C}_{7} \mathrm{H}_{5} \mathrm{ClN}_{2}(152.58)$ : C, 55.10; H, 3.30; N, 18.36. Found: C, 55.11; H, 3.32; N, 18.29.

3-Chlorophenyl cyanamide (4b). White solid; m.p. 93$95{ }^{\circ} \mathrm{C} ;{ }^{1} \mathrm{H} \mathrm{NMR}\left(\mathrm{CDCl}_{3}, 400 \mathrm{MHz}\right) \delta 6.92(\mathrm{~m}, 1 \mathrm{H}, \mathrm{ArH})$, $7.03(\mathrm{~m}, 2 \mathrm{H}, \mathrm{ArH}), 7.26(\mathrm{t}, J=8.0 \mathrm{~Hz}, 1 \mathrm{H}, \mathrm{ArH}) .{ }^{13} \mathrm{C} \mathrm{NMR}$ $\left(100 \mathrm{MHz}, \mathrm{CDCl}_{3}\right) \delta 111.1,113.8,115.9,124.0,130.9$, 135.7, 138.7. IR (KBr) 3154, 2910, 2237, 1602, 1513, 1423, $1256 \mathrm{~cm}^{-1}$. Anal. Calcd for $\mathrm{C}_{7} \mathrm{H}_{5} \mathrm{ClN}_{2}$ (152.58): C, 55.10; H, 3.30; N, 18.36. Found: C, 55.10; H, 3.29; N, 18.29. MS (ESI): $152\left(\mathrm{M}^{+}\right)$.

3-Nitrophenyl cyanamide (5b). Yellow solid; m.p. 133$135{ }^{\circ} \mathrm{C} ;{ }^{1} \mathrm{H} \mathrm{NMR}\left(\mathrm{CDCl}_{3}+\mathrm{DMSO}, 400 \mathrm{MHz}\right) \delta 7.38(\mathrm{~d}, J$ $=8.4 \mathrm{~Hz}, 1 \mathrm{H}, \operatorname{ArH}), 7.52(\mathrm{t}, J=8.4 \mathrm{~Hz}, 1 \mathrm{H}, \operatorname{ArH}), 7.85(\mathrm{~m}$, $2 \mathrm{H}, \mathrm{ArH}) .{ }^{13} \mathrm{C} \mathrm{NMR}\left(100 \mathrm{MHz}, \mathrm{CDCl}_{3}+\mathrm{DMSO}\right) \delta 109.6$, $110.7,116.8,120.8,130.1,139.9,148.4$. IR (KBr) 3147, 2919, 2241, 1621, 1531, 1354, 1260, 1071, 937, $871 \mathrm{~cm}^{-1}$. Anal. Calcd for $\mathrm{C}_{7} \mathrm{H}_{5} \mathrm{~N}_{3} \mathrm{O}_{2}$ (163.14): C, 51.54; H, 3.09; N, 25.76. Found: C, 51.58; H, 3.12; N, 25.71; MS (ESI): 163 $\left(\mathrm{M}^{+}\right)$.

4-Chlorophenyl cyanamide (6b). White solid; m.p. $95^{\circ} \mathrm{C}$; ${ }^{1} \mathrm{H}$ NMR $\left(\mathrm{CDCl}_{3}, 400 \mathrm{MHz}\right) \delta 6.91(\mathrm{~d}, J=8.0 \mathrm{~Hz}, 2 \mathrm{H}$, $\mathrm{ArH}), 7.28$ (d, $J=8.0 \mathrm{~Hz}, 2 \mathrm{H}, \mathrm{ArH}) .{ }^{13} \mathrm{C} \mathrm{NMR}(100 \mathrm{MHz}$, $\left.\mathrm{CDCl}_{3}\right) \delta 111.4,116.9,128.9,129.9,136.2 . \mathrm{IR}(\mathrm{KBr}) 3166$, 2954, 2234, 1600, 1494, 1251, $1091 \mathrm{~cm}^{-1}$. Anal. Calcd for $\mathrm{C}_{7} \mathrm{H}_{5} \mathrm{ClN}_{2}$ (152.58): C, 55.10; H, 3.30; N, 18.36. Found: C, $55.09 ; \mathrm{H}, 3.33$; N, 18.32 .

4-Methoxyphenyl cyanamide (7b). White solid; m.p. 86$89{ }^{\circ} \mathrm{C} ;{ }^{1} \mathrm{H}$ NMR $\left(\mathrm{CDCl}_{3}, 400 \mathrm{MHz}\right) \delta 3.78\left(\mathrm{~s}, 3 \mathrm{H}, \mathrm{CH}_{3}\right)$, $6.87(\mathrm{~d}, J=8.8 \mathrm{~Hz}, 2 \mathrm{H}, \mathrm{ArH}), 6.95(\mathrm{~d}, J=8.8 \mathrm{~Hz}, 2 \mathrm{H}, \mathrm{ArH})$. ${ }^{13} \mathrm{C}$ NMR $\left(100 \mathrm{MHz}, \mathrm{CDCl}_{3}\right) \delta 55.8,112.8,115.2,117.0$, 130.6, 156.1. IR (KBr) 3180, 2926, 2218, 1456, 1295, 1238, $1105,1037,826 \mathrm{~cm}^{-1}$. Anal. Calcd for $\mathrm{C}_{7} \mathrm{H}_{6} \mathrm{~N}_{2} \mathrm{O}$ (148.17): C, 64.85; H, 5.44; N, 18.91. Found: C, 64.91; H, 5.40; N, 18.93 .

4-Hydroxyphenyl cyanamide (8b). White solid; m.p. 259$261{ }^{\circ} \mathrm{C} ;{ }^{1} \mathrm{H} \mathrm{NMR}\left(\mathrm{CDCl}_{3}+\mathrm{DMSO}, 400 \mathrm{MHz}\right) \delta 5.67$ (brs, $1 \mathrm{H}, \mathrm{NH}), 6.77(\mathrm{~d}, J=8.8 \mathrm{~Hz}, 2 \mathrm{H}, \mathrm{ArH}), 6.83(\mathrm{~d}, J=8.8 \mathrm{~Hz}$, $2 \mathrm{H}, \mathrm{ArH}), 8.98$ (brs, $1 \mathrm{H}, \mathrm{OH}) .{ }^{13} \mathrm{C} \mathrm{NMR}\left(100 \mathrm{MHz} \mathrm{CDCl}_{3}\right.$ + DMSO) $\delta 112.8,115.6,115.8,129.5,152.2$. IR $(\mathrm{KBr})$ $3213,2992,2230,1613,1519,1444,1258,1224 \mathrm{~cm}^{-1}$. Anal. Calcd for $\mathrm{C}_{7} \mathrm{H}_{6} \mathrm{~N}_{2} \mathrm{O}$ (134.14): C, 62.68; H, 4.51; N, 20.88. Found: C, 62.72; H, 4.55; N, 20.83.

4-Acetylphenyl cyanamide (9b). White solid; m.p. 153$157{ }^{\circ} \mathrm{C} ;{ }^{1} \mathrm{H} \mathrm{NMR}\left(\mathrm{CDCl}_{3}+\mathrm{DMSO}, 400 \mathrm{MHz}\right) \delta 2.56(\mathrm{~s}$, 
$\left.3 \mathrm{H}, \mathrm{CH}_{3}\right), 7.08(\mathrm{~d}, J=8.8 \mathrm{~Hz}, 2 \mathrm{H}, \mathrm{ArH}), 7.91(\mathrm{~d}, J=8.8 \mathrm{~Hz}$, $2 \mathrm{H}, \mathrm{ArH}) .{ }^{13} \mathrm{C} \mathrm{NMR}\left(100 \mathrm{MHz}, \mathrm{CDCl}_{3}+\mathrm{DMSO}\right) \delta 25.9$, $110.9,114.5,129.8,131.2,142.9,196.2$. IR (KBr) 3188, 2966, 2228, 1666, 1599, 1585, 1411, 1362, 1278, 1176, 962 $\mathrm{cm}^{-1}$. Anal. Calcd for $\mathrm{C}_{9} \mathrm{H}_{8} \mathrm{~N}_{2} \mathrm{O}$ (160.18): C, 67.49; $\mathrm{H}, 5.03$; $\mathrm{N}, 17.48$. Found: C, 67.53; H, 5.08; N, 17.44. MS (ESI): 160 $\left(\mathrm{M}^{+}\right)$.

2-Iodo-4-methylphenyl cyanamide (10b). White solid; m.p. $144{ }^{\circ} \mathrm{C}$; ${ }^{1} \mathrm{H} \mathrm{NMR}\left(\mathrm{CDCl}_{3}, 400 \mathrm{MHz}\right) \delta 2.29(\mathrm{~s}, 3 \mathrm{H}$, $\mathrm{CH}_{3}$ ), 6.17 (brs, $\left.1 \mathrm{H}, \mathrm{NH}\right), 7.17$ (dd, $\left.J=8.2 \mathrm{~Hz}, 2 \mathrm{H}, \mathrm{ArH}\right)$, 7.56 (s, $1 \mathrm{H}, \mathrm{ArH}) .{ }^{13} \mathrm{C} \mathrm{NMR}\left(100 \mathrm{MHz}, \mathrm{CDCl}_{3}\right) \delta 20.4$, 84.2, 110.7, 115.4, 130.9, 135.4, 139.6. IR (KBr) 3229, 2919, 2217, 1603, 1573, 1502, 1420, 1383, 1283, 1032, 866, 805 $\mathrm{cm}^{-1}$. Anal. Calcd for $\mathrm{C}_{8} \mathrm{H}_{7} \mathrm{~N}_{2}$ (258.06): C, 37.23; H, 2.73; N, 10.86. Found: C, 37.27; H, 2.75; N, 10.84.

n-Butyl cyanamide (11b). Gummy; ${ }^{1} \mathrm{H}$ NMR $\left(\mathrm{CDCl}_{3}, 400\right.$ $\mathrm{MHz}) \delta 0.94\left(\mathrm{t}, J=7.6 \mathrm{~Hz}, 3 \mathrm{H}, \mathrm{CH}_{3}\right), 1.40\left(\mathrm{~m}, 2 \mathrm{H}, \mathrm{CH}_{2}\right)$, $1.58\left(\mathrm{~m}, 2 \mathrm{H}, \mathrm{CH}_{2}\right), 3.06\left(\mathrm{~m}, 2 \mathrm{H}, \mathrm{CH}_{2}\right), 4.61$ (brs, $\left.1 \mathrm{H}\right) .{ }^{13} \mathrm{C}$ NMR $\left(100 \mathrm{MHz} \mathrm{CDCl}_{3}\right) \delta 13.6,19.5,31.7,45.7,117.2$. IR (KBr) 3207, 2961, 2875, 2221, 1614, 1463, 1373, $1171 \mathrm{~cm}^{-1}$. Anal. Calcd for $\mathrm{C}_{5} \mathrm{H}_{10} \mathrm{~N}_{2}$ (98.15): C, 61.19; H, 10.27; N, 28.54. Found: C, 61.22; H, 10.23; N, 28.48.

Cyclohexyl cyanamide (12b). Gummy; ${ }^{1} \mathrm{H} \mathrm{NMR}\left(\mathrm{CDCl}_{3}\right.$, $400 \mathrm{MHz}) \delta 1.31\left(\mathrm{~m}, 5 \mathrm{H}, \mathrm{CH}_{2}\right), 1.61\left(\mathrm{~m}, 1 \mathrm{H}, \mathrm{CH}_{2}\right), 1.78$ $\left(\mathrm{m}, 2 \mathrm{H}, \mathrm{CH}_{2}\right), 1.95\left(\mathrm{~m}, 2 \mathrm{H}, \mathrm{CH}_{2}\right), 3.09\left(\mathrm{~m}, 1 \mathrm{H}, \mathrm{CH}_{2}\right), 3.91$ (brs, $1 \mathrm{H}, \mathrm{NH}) .{ }^{13} \mathrm{C} \mathrm{NMR}\left(100 \mathrm{MHz}, \mathrm{CDCl}_{3}\right) \delta 24.3,25.1$, 32.6, 54.3, 115.9. IR (KBr) 3196, 2933, 2857, 2217, 1453, 1367, $1167 \mathrm{~cm}^{-1}$. Anal. Calcd for $\mathrm{C}_{7} \mathrm{H}_{12} \mathrm{~N}_{2}$ (124.19): C, 67.70; H, 9.74; N, 22.56. Found: C, 67.67; H, 9.70; N, 22.50.

Benzyl cyanamide (13b). Gummy; ${ }^{1} \mathrm{H} \mathrm{NMR}\left(\mathrm{CDCl}_{3}, 400\right.$ $\mathrm{MHz}) \delta 4.11\left(\mathrm{~d}, J=5.2 \mathrm{~Hz}, 2 \mathrm{H}, \mathrm{CH}_{2} \mathrm{CH}_{2}\right), 4.66$ (brs, $1 \mathrm{H}$, $\mathrm{NH}), 7.27-7.37(\mathrm{~m}, 5 \mathrm{H}, \mathrm{ArH}) .{ }^{13} \mathrm{C}$ NMR (100 MHz, $\left.\mathrm{CDCl}_{3}\right) \delta 49.9,116.7,127.9,128.4,128.9,136.4$. IR (KBr) $3207,2925,2220,1455,1359,1155,1014 \mathrm{~cm}^{-1}$. Anal. Calcd for $\mathrm{C}_{8} \mathrm{H}_{8} \mathrm{~N}_{2}$ (132.17): C, 72.70; $\mathrm{H}, 6.10 ; \mathrm{N}, 21.19$. Found: C, 72.66; H, 6.13; N, 21.11 .

Benzo[1,3] dioxol-5-ylmethyl cyanamide (14b). White solid; m.p. 82-84 ${ }^{\circ} \mathrm{C}$; ${ }^{1} \mathrm{H}$ NMR $\left(\mathrm{CDCl}_{3}, 400 \mathrm{MHz}\right) \delta 4.05$ (d, $J=5.2 \mathrm{~Hz}, 2 \mathrm{H}, \mathrm{CH}_{2}$ ), 4.57 (brs, $\left.1 \mathrm{H}, \mathrm{NH}\right), 5.94$ (s, 2H, $\left.\mathrm{OCH}_{2}\right), 6.77(\mathrm{~m}, 3 \mathrm{H}) .{ }^{13} \mathrm{C} \mathrm{NMR}\left(100 \mathrm{MHz}, \mathrm{CDCl}_{3}\right) \delta 49.9$, $101.4,108.46,108.54,116.5,121.7,130.1,147.8,148.2$. IR (KBr) 3233, 2952, 2897, 2220, 1500, 1445, 1038, 925, 809 $\mathrm{cm}^{-1}$. Anal. Calcd for $\mathrm{C}_{9} \mathrm{H}_{8} \mathrm{~N}_{2} \mathrm{O}_{2}$ (176.18): C, 61.36; $\mathrm{H}, 4.58$; N, 15.90. Found: C, 61.41; H, 4.61; N, 15.85 .

3,4-Dimethoxyphenylethyl cyanamide (15b). Gummy; ${ }^{1} \mathrm{H} \mathrm{NMR}\left(\mathrm{CDCl}_{3}, 400 \mathrm{MHz}\right) \delta 2.84\left(\mathrm{t}, 2 \mathrm{H}, \mathrm{CH}_{2}\right), 3.28(\mathrm{q}, \mathrm{J}$ $\left.=7.2 \mathrm{~Hz}, 2 \mathrm{H}, \mathrm{CH}_{2}\right), 3.83\left(\mathrm{~s}, 3 \mathrm{H}, \mathrm{CH}_{3}\right), 3.84\left(\mathrm{~s}, 3 \mathrm{H}, \mathrm{CH}_{3}\right)$, 4.37 (brs, $1 \mathrm{H}, \mathrm{NH}), 6.76(\mathrm{~m}, 3 \mathrm{H}, \mathrm{ArH}) .{ }^{13} \mathrm{C}$ NMR $(100$ $\left.\mathrm{MHz}, \mathrm{CDCl}_{3}\right) \delta 35.5,47.5,55.92,55.95,111.4,111.9,116.5$, $120.9,130.0,147.8,148.9$. IR (KBr) 3274, 2937, 2219, 1592,
1517, 1464, 1262, 1236, 1156, 1142, 1026, $913 \mathrm{~cm}^{-1}$. Anal. Calcd for $\mathrm{C}_{11} \mathrm{H}_{14} \mathrm{~N}_{2} \mathrm{O}_{2}(206.24)$ : C, 64.06; H, 6.84; N, 13.58 . Found: C, 64.12; H, 6.80; N, 13.54.

\section{Conclusion}

In conclusion, we have developed a general, economical and environmentally benign method for the preparation of isothiocyanates and cyanamides from their corresponding dithiocarbamic acid salts. The use of non-toxic and eco-friendly reagents and solvents without the formation of any side products makes this methodology potentially useful. The yield could in fact be considered as very good if not excellent.

Acknowledgements UBS acknowledges the financial support received from UGC (UGC/MRP 43-192/ 2014). N. B. Kuotsu acknowledges the support of this research from UGC (F.5.77/2014-15/MRP/NERO) Thanks are due to CIF/IIT Guwahati for NMR spectra

\section{References}

1. (a) A. K. Mukerjee, R. Ashare, Chem. Rev. 1991, 91, 1-24. DOI:10.1021/cr00001a001

(b) M. Dobosz, M. Wujec, Heterocycles, 2002, 57, 1135-1141. DOI: $10.3987 / C O M-02-9461$

2. (a) N. Kuhnert, G. Williamson, B. Holst, J. Labl. Comp. Radiopharm. 2001, 44, 347-355. DOI:10.1002/jlcr.462

(b) N. Kuhnett, Y. Lu, Labl. Comp. Radiopharm. 2004, 47, 501-507. DOI:10.1002/jlcr.833

3. C. Nastruzzi, R. Cotesi, E. Eposito, E. Menegatti, O. Leoni, R. Ironi, S. J. Palmier, Agric. Food. Chem. 2000, 48, 3572-3575. DOI:10.1021/jf000191p

4. K. Xu, P. Thornalley, J. Biochem. Pharmacol. 2000, 6, 221-231. DOI:10.1016/S0006-2952(00)00319-1

5. C. X. Zhang, K. Y. Lee, A. J. Kelly, R. T. Burke, J. Org. Chem. 2000, 65, 6237-6240. DOI:10.1021/jo000139s

6. (a) J. E. Hodgkins, W. P. Reeves, J. Org. Chem. 1956, 21, 404405. DOI:10.1021/jo01110a006

(b) J. E. Hodgkins, W. P. Reeves, J. Org. Chem. 1964, 29, 30983099. DOI:10.1021/jo01033a524

7. C. G. Cho, G. H. Posne, Tetrahedron Lett. 1992, 33, 35993602. DOI:10.1016/S0040-4039(00)92512-7

8. T. Kitamura, S. Kobayashi, H. Taniguchi, J. Org. Chem. 1990, 55, 1801-1805. DOI:10.1021/jo00293a025

9. J. N. Kim, K. S. Jung, J. H. Lee, J. S. Son, Tetrahedron Lett. 1997, 38, 1597-1598. DOI:10.1016/S0040-4039(97)00121-4

10. J. Goerdeler, C. Ho, Chem. Ber. 1984, 117, 1636-1639. DOI:10.1002/cber.19841170431

11. H. M. Mesheram, S. Dale, J. S. Yadav, Tetrahedron Lett. 1997, 38, 8743-8744. DOI:10.1016/S0040-4039(97)10158-7

12. A. Toshimitsu, S. Uemura, M. Okano, N. Watanabe, J. Org. Chem. 1983, 48, 5246-5251. DOI:10.1021/jo00174a018

13. D. Albanese, M. Penso, Synthesis 1991, 1001-1002. 


\section{DOI:10.1055/s-1991-26629}

14. W. G. Shan, G. F. Bian, W. K. Su, X. R. Liang, Org. Prep. Proced. Int. 2004, 36, 283-286.

DOI:10.1080/00304940409355967

15. J. N. Kim, E. K. Ryu, Tetrahedron Lett. 1993, 34, 8283-8284. DOI:10.1016/S0040-4039(00)61411-9

16. J. N. Kim, K. S. Jung, H. J. Lee, J. S. Son, Tetrahedron Lett. 1997, 38, 1597-1598. DOI:10.1016/S0040-4039(97)00121-4

17. W. Adam, R. M. Bargon, S. G. Bosio, W. A. Schenk, D. Stalke, J. Org. Chem. 2002, 67, 7037-7041. DOI:10.1021/jo026042i

18. M. Arisawa, M. Ashikawa, A. Suwa, M. Yamaguchi, Tetrahedron Lett. 2005, 46, 1727-1729.

DOI:10.1016/j.tetlet.2005.01.069

19. L. Valette, S. Poulain, X. Fernandez, L. Lizzani-Cuvelier, J. Sulfur Chem. 2005, 26, 155-161.

DOI:10.1080/17415990500070144

20. T. Isoda, K. Hayashi, S. Tamai, T. Kumagai, Y. Nagao, Chem. Pharm. Bull. 2006, 54, 1616-1619. DOI:10.1248/cpb.54.1616

21. B. Zhong, R. S. Al-Awar, C. Shih, J. H. Grimes Jr., M. Vieth, C. Hamdouchi, Tetrahedron Lett. 2006, 47, 2161-2164.

DOI:10.1016/j.tetlet.2006.01.119

22. W. J. Neely, Aust. J. Chem. 1960, 13, 341-346.

DOI:10.1071/CH9600341

23. M. Bollini, J. J. Casal, D. E. Alvarez, L. Boiani, M. González, H. Cerecetto, A. M. Bruno, Bioorg. Med. Chem. 2009, 17, 1437-1444. DOI:10.1016/j.bmc.2009.01.011

24. E. Dyer, T. B. Johnson, J. Am. Chem. Soc. 1932, 54, 777-787. DOI:10.1021/ja01341a048

25. G. M. Dyson, T. Harrington, J. Chem. Soc. 1942, 374-375. DOI:10.1039/JR9420000374

26. J. C. Jochims, A. Seeliger, Tetrahedron 1965, 21, 2611-2616. DOI:10.1016/S0040-4020(01)93917-1

27. R. Gottfried, Angew. Chem., Int. Ed. Engl. 1966, 5, 963-964. DOI:10.1002/anie.196609632

28. C. Larsen, K. Steliou, D. N. Harpp, J. Org. Chem. 1978, 43, 337-339. DOI:10.1021/jo00396a035

29. C. Larsen, D. N. Harpp, J. Org. Chem. 1981, 46, 2465-2466. DOI:10.1021/jo00325a007

30. S. Kim, K. Y. Yi, J. Org. Chem. 1986, 51, 2613-2615. DOI:10.1021/jo00363a046

31. S. Kim, K. Y. Yi, Tetrahedron Lett. 1985, 26, 1661-1664. DOI:10.1016/S0040-4039(00)98578-2

32. J. Grayson, I. Org. Process Res. Dev. 1997, 1, 240-246. DOI:10.1021/op970002c

33. (a) A. Servais, M. Azzouz, D. Lopes, C. Courillon, M. Malacria, Angew. Chem. Int. Ed. 2007, 46, 576-579.

DOI:10.1002/anie.200602940

(b) M. H. Larraufie, C. Ollivier, L. Fensterbank, M. Malacria, E. Laocte, Angew. Chem. Int. Ed. 2010, 49, 2178-2181. DOI:10.1002/anie.200907237

(c) Z. Pan, S. M. Pound, N. R. Rondla, C. J. Douglas, Angew. Chem., Int. Ed. 2014, 53, 5170-5174.

(d) H. Basavaprabhu, V. V. Sureshbabu, Org. Biomol. Chem. 2012, 10, 2528-2533. DOI:10.1039/c2ob06916d

(e) V. Panduranga, H. Basavaprabhu, V. V. Sureshbabu, Tetrahedron Lett. 2013, 54, 975-979.
DOI:10.1016/j.tetlet.2012.12.027

(f) S. Kamijo, T. Jin, Y. Yamamoto, Angew. Chem. Int. Ed. 2002, 41, 1780-1782. DOI:10.1002/1521-3773(20020517) 41:10<1780::AID-ANIE1780>3.0.CO;2-\#

(g) L. V. R. Bonaga, H. C. Zhang, B. E. Maryanoff, Chem. Commun. 2004, 2394-2395. DOI:10.1039/B410012C

(h) K. Fukumoto, T. Oya, M. Itazaki, H. Nakazawa, J. Am. Chem. Soc. 2009, 131, 38-39. DOI:10.1021/ja807896b

(i) R. L. Giles, J. D. Sullivan, A. M. Steiner, R. E. Looper, Angew. Chem., Int. Ed. 2009, 48, 3116-3120.

DOI:10.1002/anie.200900160

(j) S. Guin, S. K. Rout, A. Gogoi, W. Ali, B. K. Patel, Adv. Synth. Catal. 2014, 356, 2559-2565.

DOI:10.1002/adsc.201400011

(k) T. K. Lane, B. R. D’Souza, J. Louie, J. Org. Chem. 2012, 77, 7555-7563. (l) V. Kumar, M. P. Kaushik, A. Mazumdar, Eur. J. Org. Chem. 2008, 1910-1916. DOI:10.1021/jo3012418

34. (a) C. J. Adams, J. Chem. Soc., Dalton Trans. 1999, 2059-2064. DOI:10.1039/a901959f

(b) A. S. Smimov, E. S. Butukhanova, N. A. Bokach, G. L. Starova, V. V. Gurzhiy, M. L. Kuznetsov, V. Y. Kukushkin, Dalton Trans. 2014, 43, 15798-15811.

DOI:10.1039/C4DT01812E

(c) M. Yuan, S. Gao, H. L. Sun, G. Su, Inorg. Chem. 2004, 43, 8221-8223. DOI:10.1021/ic048547s

35. (a) D. G. Barret, D. N. Deaton, A. M. Hassell, R. B. McFadyen, A. B. Miller, L. R. Miller, J. A. Payne, L. M. Shewchuk, D. H. Willard, L. L. Wright, Bioorg. Med. Chem. Lett. 2005, 15, 3039-3043. DOI:10.1016/j.bmcl.2005.04.032

(b) H. W. K. Bischofsheim, H. J. L. Hogheim, J. R. S. Kelkheim, A. W. Egelsbach, S. F. Idstein, H. W. J. Niedernhausen, S. P. Frankfurt, US 6369069B1, 2002.

(c) K. S. Atwal, G. J. Grover, S. Z. Ahmed, P. G. Sleph, S. Dzwonczyk, A. J. Baird, D. E. Normandin, J. Med. Chem. 1995, 38, 3236-3245. DOI:10.1021/jm00017a007

(d) P. W. Manley, U. Quast, J. Med. Chem. 1992, 35, 23272340. DOI:10.1021/jm00090a025

(e) G. Danger, A. Michaut, M. Bucchi, L. Boiteau, J. Canal, R. Plascal, Angew. Chem. Int. Ed. 2013, 52, 611-614.

DOI:10.1002/anie.201207730

36. T. Kamo, M. Endo, M. Sato, R. Kasahara, H. Yamaya, S. Hiradate, Y. Fujii, N. Hirai, M. Hirota, Phytochemistry 2008, 69, 1166-1172. DOI:10.1016/j.phytochem.2007.11.004

37. (a) B. J. Von, Ber. Dtsch. Chem. Ges. 1900, 33, 1438-1452. DOI:10.1002/cber.19000330208

(b) L. Y. Hu, J. Guo, S. S. Mager, J. B. Fischer, K. J. Burke-Howie, G. J. Durant, Ber. Dtsch. Chem. Ges. 1900, 33, 1438-1452. DOI: $10.1002 /$ cber. 19000330208

(c) G. Kaupp, J. Schmeyers, J. Boy, Chem.-Eur. J. 1998, 4, 24672474. DOI:10.1002/(SICI)1521-3765(19981204)4:12<2467: :AID-CHEM2467>3.0.CO;2-D

38. (a) W. A. Davis, M. P. Cava, J. Org. Chem. 1983, 48, 27742775. DOI:10.1021/jo00164a030

(b) D. Kahne, D. Collum, Tetrahedron Lett. 1981, 22, 50115014. DOI:10.1016/S0040-4039(01)92406-2

39. K. H. Boltz, H. D. Dell, Justus Liebigs Ann. Chem. 1967, 709, 
63-67. DOI:10.1002/jlac.19677090107

40. M. E. Hermes, F. D. Marsh, J. Org. Chem. 1972, 37, 29692979. DOI: $10.1021 /$ jo00984a013

41. T. V. Hughes, S. D. Hammond, M. P. Cava, J. Org. Chem. 1998, 63, 401-402. DOI:10.1021/jo9717548

42. R. C. Wheland, E. L. Martin, J. Org. Chem. 1975, 40, 31013109. DOI:10.1021/jo00909a019

43. Y. Q. Wu, D. C. Limburg, D. E. Wilkinson, G. S. Hamilton, Org. Lett. 2000, 2, 795-297. DOI:10.1021/ol0055263

44. J. J. Kim, D. J. Kweon, S. D. Cho, K. H. Kim, E.Y. Jung, S. G. Lee, J. R. Falck, Y. J. Yoon, Tetrahedron 2005, 61, 5889-5894. DOI:10.1016/j.tet.2005.03.138

45. S. A. Bakunov, A. V. Rukavishnikov, A. V. Tkachev, Synthesis 2000, 1148-1159. DOI:10.1055/s-2000-6317
46. K. Shin, J. Tienan, Y. Yoshinori, J. Am. Chem. Soc. 2001, 123, 9453-9454. DOI:10.1021/ja016355f

47. (a) F. F. Wong, C. Y. Chen, M. Y. Yeh, Synlett 2006, 559-562. DOI:10.1055/s-2006-932470

(b) C. Y. Chen, F. F. Wong, J. J. Haung, S. K. Lin, M. Y. Yeh, Tetrahedron Lett. 2008, 49, 6505-6507.

DOI:10.1016/j.tetlet.2008.08.106

48. K. H. Chaudhuri, U. S. Mahajan, D. S. Bhalerao, K. G. Akamanchi, Synlett 2007, 2815-2818.

49. J. Nath, B. Patel, L. Jamir, U. B. Sinha, K. V. V. V. Satyanarayana, Green Chem. 2009, 11, 1503-1506.

DOI:10.1039/b914283p

\section{Povzetek}

Opisujemo zelo učinkovit in enostaven pristop k sintezi izotiocianatov in cianamidov iz ustreznih aminov ob prisotnosti blagega, učinkovitega in nestrupenega reagenta tetrapropilamonijevega tribromida. Dobra okoljska sprejemljivost, stroškovna učinkovitost in visoki izkoristki so glavne odlike te metodologije. 